\title{
Exact greybody factors for the brane scalar field of five-dimensional rotating black holes
}

\author{
Young-Hwan Hyun, ${ }^{a}$ Yoonbai $\mathrm{Kim}^{b}$ and Seong Chan Park \\ ${ }^{a}$ Korea Institute of Science and Technology Information (KISTI), \\ 245 Daehak-ro, Yuseong-gu, Daejeon 34141, Republic of Korea \\ ${ }^{b}$ Department of Physics and Institute of Basic Science, Sungkyunkwan University, \\ Suwon 16419, Republic of Korea \\ ${ }^{c}$ Department of Physics and IPAP, Yonsei University, \\ Seoul 03722, Republic of Korea \\ E-mail: younghwan.hyun@gmail.com, yoonbai@skku.edu, \\ sc.park@yonsei.ac.kr
}

ABSTRACT: We study scalar perturbations of the five-dimensional rotating black holes and find an exact solution giving exact description of the Hawking radiation. Mathematically, the full solution for this spin-zero field is expressed in terms of the prolate spheroidal wave function with complex parameters. By using the spheroidal joining factor, we write the corresponding boundary condition and greybody factors. We also check that the exact result reproduces the low frequency limit of the greybody factor and shows good agreement with the known numerical results.

Keywords: Phenomenology of Field Theories in Higher Dimensions, Phenomenology of Large extra dimensions

ARXIV EPRINT: 1901.10828 


\section{Contents}

1 Introduction 1

2 Generalized Teukolsky equations on brane 2

3 Exact solutions of generalized Teukolsky equations 4

4 Boundary condition near the horizon $\quad 7$

5 Greybody factors for scalar fields $\quad 9$

6 Summary and discussions 11

A Myers-Perry black holes $\quad 12$

$\begin{array}{ll}\text { B Spheroidal equation } & 13\end{array}$

\section{Introduction}

Higher dimensional black holes have been attractive objects which may shed light on understanding extra dimensions. A particular interest has been received for the low-scale gravity scenario [1-5] due to a possibility to be produced in proton-proton collisions at the LHC $[6,7]$ and comic ray-nucleon collisions at the atmosphere of the earth [8-11]. (See the comprehensive reviews [12-14].)

In the low energy gravity scenarios, the fundamental gravity scale is as low as the electroweak scale, $M_{*} \sim \mathcal{O}(1) \mathrm{TeV}$, and thus the trans-Planckian particle collision with the collision energy, $\sqrt{s} \gg M_{*}$, generates higher dimensional black holes with a mass larger than the Planck scale, $M_{\mathrm{bh}} \gg M_{*}$. In this paradigm, classical laws of black hole physics still hold as long as the black hole is larger than the Planck length, $r_{\mathrm{bh}} \gg 1 / M_{*}$. After the production of the black holes with a mass larger than the Planck scale, it is anticipated to decay via emitting Hawking radiation [15]. During the spin-down and Schwarzschild phase, the produced black hole radiates particles and loses its mass until the mass becomes of Planck scale where the Hawking radiation is not applicable in the present form. This process allows to test whether the extra-dimensions exist or not and to see which low scale gravity model gives us good approximations. For this reason, studying the higher dimensional, rotating black holes in detail is getting important.

To identify the signal of any higher dimensional black hole, the study on the propagation of the particles from the black holes must be preceded. According to some of the low scale gravity scenarios [1-5], gravitons propagate in the bulk, while the standard model particles are confined to live on a $(1+3)$-dimensional brane. The black holes radiate mainly 
on the brane [16]. The propagation of these perturbations is governed by the Teukolsky equation [17] in the background of a higher-dimensional, rotating black hole. Once the Teukolsky equation is given [18], a cornerstone is to find the exact solution which enables us various relevant analyses including the Hawking radiation and the stability of the black holes. However, finding an exact solution of the Teukolsky equation is tough and thus previous investigations assumed a low-frequency regime [18, 21-23] or only used numerical methods [19, 20, 24-27]. Overcoming these restrictions and finding the analytic, exact expressions for the greybody factor for the higher dimensional, rotating black hole is extremely important but never has been successful. Only limited results for the non-rotating black hole are found for spherically symmetric, static black hole in $d$ dimension [28]. In this work, we could make some partial success in finding the exact solutions: the fully analytic results for the scalar mode on the brane without the low frequency restriction are found! Previously known analytic and numerical results are successfully re-driven from our results.

This paper is composed as follows: in section 2 and 3, we find the general $s=0$ solution of the Teukolsky equation on the brane in an exact form. In section 4 , the boundary conditions are assigned, appropriate for the ingoing waves. In section 5 , the exact ingoing wave solution is applied to compute the greybody factors and the obtained exact formulas are compared to the approximate results. We conclude the section 6 with discussions.

\section{Generalized Teukolsky equations on brane}

We consider a scalar field in background of the 5D Myers-Perry black holes. The scalar field propagates on the four-dimensional brane and obeys the linear equation coupled to the background gravity. In this section, by use of separation of the variables and subsequently by solving some part of the obtained ordinary differential equations, we show that the resultant decoupled ordinary differential equations become uniquely the generalized Teukolsky equations of $s=0$.

We begin our discussion with the introduction of a real scalar field $\phi(x)$ whose dynamics is governed by the Klein-Gordon equation,

$$
\nabla^{2} \phi=\frac{1}{\sqrt{-g}} \partial_{\mu}\left(\sqrt{-g} g^{\mu \nu} \partial_{\nu} \phi\right)=0 .
$$

This scalar field $\phi$ propagates on the four-dimensional brane in the five-dimensional bulk formed by the Myers-Perry black hole [29] whose metric is expressed in the Boyer-Lindquist coordinates as

$$
\begin{aligned}
d s^{2}= & \left(1-\frac{\tilde{\mu}}{\Sigma(r, \theta)}\right) \mathrm{d} t^{2}+2 a \sin ^{2} \theta \frac{\tilde{\mu}}{\Sigma(r, \theta)} \mathrm{d} t \mathrm{~d} \phi-\sin ^{2} \theta\left(r^{2}+a^{2}+a^{2} \sin ^{2} \theta \frac{\tilde{\mu}}{\Sigma(r, \theta)}\right) \mathrm{d} \phi^{2} \\
& -\frac{\Sigma(r, \theta)}{\Delta(r)} \mathrm{d} r^{2}-\Sigma(r, \theta) \mathrm{d} \theta^{2}-r^{2} \cos ^{2} \theta \mathrm{d} \psi^{2},
\end{aligned}
$$

where the functions in the metric have

$$
\begin{aligned}
\Sigma(r, \theta) & =r^{2}+a^{2} \cos ^{2} \theta, \\
\Delta(r) & =r^{2}+a^{2}-\tilde{\mu} \equiv r^{2}-r_{\mathrm{h}}^{2} .
\end{aligned}
$$


For the Myers-Perry black hole, see appendix A. The parameters $\tilde{\mu}$ and $a$ are a normalized mass and a normalized angular momentum, respectively. From $\Delta\left(r_{\mathrm{h}}\right)=0$, we read an event horizon at $r=r_{\mathrm{h}}$. Thus the induced metric for the brane scalar field in four dimensions is obtained by neglecting $\psi$ dependence, $\mathrm{d} \psi=0$ in (2.2), and, in this background gravity, the brane scalar field follows the linear equation from (2.1),

$$
\begin{gathered}
{\left[\left(1+\frac{\mu\left(r^{2}+a^{2}\right)}{\Delta \Sigma}\right) \frac{\partial^{2}}{\partial t^{2}}-\frac{1}{\Sigma} \frac{\partial}{\partial r} \Delta \frac{\partial}{\partial r}-\frac{1}{\Sigma \sin \theta} \frac{\partial}{\partial \theta} \sin \theta \frac{\partial}{\partial \theta}\right.} \\
\left.-\frac{1}{\Delta \sin ^{2} \theta}\left(1-\frac{\mu}{\Sigma}\right) \frac{\partial^{2}}{\partial \varphi^{2}}+\frac{2 a \mu}{\Delta \Sigma} \frac{\partial^{2}}{\partial t \partial \varphi}\right] \phi=0 .
\end{gathered}
$$

Separation of variables $\phi \equiv T(t) R(r) Y(\theta, \varphi)$ to the equation (2.5) results in

$$
\frac{\mathrm{d}^{2} T(t)}{\mathrm{d} t^{2}}+f(r, \theta, \varphi) \frac{\mathrm{d} T(t)}{\mathrm{d} t}+g(r, \theta, \varphi) T(t)=0
$$

where $f$ and $g$ are time-independent but involve both the spatial variables $(r, \theta, \varphi)$ and their derivatives as

$$
\begin{gathered}
f=\left(1+\frac{\mu\left(r^{2}+a^{2}\right)}{\Delta \Sigma}\right)^{-1} \frac{2 a \mu}{\Delta \Sigma} \frac{1}{Y(\theta, \varphi)} \frac{\partial Y(\theta, \varphi)}{\partial \varphi}, \\
g=-\left(1+\frac{\mu\left(r^{2}+a^{2}\right)}{\Delta \Sigma}\right)^{-1}\left[\frac{1}{\Sigma} \frac{1}{R(r)} \frac{\mathrm{d}}{\mathrm{d} r}\left(\Delta \frac{\mathrm{d} R(r)}{\mathrm{d} r}\right)+\frac{1}{\Sigma \sin \theta} \frac{1}{Y(\theta, \varphi)} \frac{\partial}{\partial \theta}\left(\sin \theta \frac{\partial Y(\theta, \varphi)}{\partial \theta}\right)\right. \\
\left.\quad+\frac{1}{\Delta \sin ^{2} \theta}\left(1-\frac{\mu}{\Sigma}\right) \frac{1}{Y(\theta, \varphi)} \frac{\partial^{2} Y(\theta, \varphi)}{\partial \varphi^{2}}\right] .
\end{gathered}
$$

The time-dependent part $T(t)$ of the linear equation (2.6) is solved by the general solution,

$$
T(t)=A_{+} e^{-i \omega_{+} t}+A_{-} e^{-i \omega_{-} t},
$$

where $\omega_{ \pm}=-\frac{i}{2}\left(f \pm \sqrt{f^{2}-4 g}\right)$. Since $T$ depends only on time $t$ for both non-zero coefficients $A_{ \pm}$, constancy of the two frequencies $\omega_{ \pm}$requires both $f$ and $g$ to be constants independent of other variables $(r, \theta, \varphi)$. Further separation of variables, $Y(\theta, \varphi) \equiv S(\theta) \Phi(\varphi)$, reduces the equation (2.7) to the equation of $\Phi$,

$$
\frac{\partial \Phi(\varphi)}{\partial \varphi}=i \frac{\omega_{+}+\omega_{-}}{2 a \mu}\left[\Delta \Sigma+\mu\left(r^{2}+a^{2}\right)\right] \Phi(\varphi)
$$

where $f$ is replaced by $\omega_{ \pm}$. Since the functions in front of $\Phi(\varphi)$ in the right-hand side does not depend on the variable $\varphi$, its solution up to an overall constant is given with a constant $m$ by

$$
\Phi(\varphi)=e^{i m \varphi}, \quad(m \in \mathbb{Z})
$$

which leads to a constraint,

$$
\frac{\Delta \Sigma+\mu\left(r^{2}+a^{2}\right)}{2 a \mu}\left(\omega_{+}+\omega_{-}\right)=m
$$


Since this algebraic equation can become meaningful only for $m=0$ and $\omega_{+}+\omega_{-}=0$, the constant $f$ in (2.7) is given by zero and thus, from (2.6) $\varphi$-dependence should disappear, $Y(\theta, \varphi)=S(\theta)$.

Let us consider another case of $A_{+}=0$ or $A_{-}=0$. Then the solutio has $T(t)=e^{-i \omega t}$ with constant $\omega$ of $2 i \omega=f \pm \sqrt{f^{2}-4 g}$. Further separation of variables, $Y(\theta, \varphi) \equiv S(\theta) \Phi(\varphi)$, makes the equation be

$$
\frac{\mathrm{d}^{2} \Phi(\varphi)}{\mathrm{d} \varphi^{2}}+p(r, \theta) \frac{\mathrm{d} \Phi(\varphi)}{\mathrm{d} \varphi}+q(r, \theta) \Phi(\varphi)=0,
$$

where the two functions $p(r, \theta)$ and $q(r, \theta)$ are

$$
\begin{aligned}
p(r, \theta) & =\frac{2 a \mu \omega \sin ^{2} \theta}{\Sigma-\mu}, \\
q(r, \theta)= & \frac{\Delta \sin ^{2} \theta}{\Sigma-\mu}\left[\frac{1}{R(r)} \frac{\mathrm{d}}{\mathrm{d} r}\left(\Delta \frac{\mathrm{d} R(r)}{\mathrm{d} r}\right)+\frac{1}{\sin \theta} \frac{1}{S(\theta)} \frac{\mathrm{d}}{\mathrm{d} \theta}\left(\sin \theta \frac{\mathrm{d}}{\mathrm{d} \theta} S(\theta)\right)\right. \\
& \left.-\omega^{2}\left(\Sigma+\frac{\mu\left(r^{2}+a^{2}\right)}{\Delta}\right)\right] .
\end{aligned}
$$

In the same manner, the solution of $\Phi(\varphi)$ can takes the form,

$$
\Phi(\varphi)=A_{+}^{\prime} e^{i m_{+} \varphi}+A_{-}^{\prime} e^{i m_{-} \varphi},
$$

where $m_{ \pm}=\frac{i}{2}\left(p \pm \sqrt{p^{2}-4 q}\right)$. The coordinate dependence of $p(r, \theta)$ again restricts the coefficients as $A_{+}^{\prime}=0$ or $A_{-}^{\prime}=0$, that leads to (2.11) with an arbitrary integer $m$. The straightforward calculation results in the generalized Teukolsky equation for brane scalar fields,

$$
\begin{array}{r}
\frac{1}{\sin \theta} \frac{\mathrm{d}}{\mathrm{d} \theta}\left(\sin \theta \frac{\mathrm{d}}{\mathrm{d} \theta} S(\theta)\right)+\left[(a \omega \cos \theta)^{2}-(m \csc \theta)^{2}+A\right] S(\theta)=0, \\
\frac{\mathrm{d}}{\mathrm{d} r}\left(\Delta \frac{\mathrm{d}}{\mathrm{d} r} R(r)\right)+\left[\frac{K^{2}}{\Delta}+2 m a \omega-a^{2} \omega^{2}-A\right] R(r)=0,
\end{array}
$$

where $A$ is a separation constant and $K(r)=\left(r^{2}+a^{2}\right) \omega-m a$. Note that the previous case is nothing but the spacial case of $m=0$ case in this solution.

\section{Exact solutions of generalized Teukolsky equations}

The generalized Teukolsky equation for the brane fields in the 5D spacetime is obtained by applying the Newman-Penrose formalism to the four-dimensional induced metric [18]. Then the perturbation equations are given as separated differential equations with a separation constant $A$,

$$
\begin{array}{r}
\frac{1}{\sin \theta} \frac{\mathrm{d}}{\mathrm{d} \theta}\left(\sin \theta \frac{\mathrm{d} S}{\mathrm{~d} \theta}\right)+\left[(s-a \omega \cos \theta)^{2}-(s \cot \theta+m \csc \theta)^{2}-s(s-1)+A\right] S=0, \\
\Delta^{-s} \frac{\mathrm{d}}{\mathrm{d} r}\left(\Delta^{s+1} \frac{\mathrm{d} R}{\mathrm{~d} r}\right)+\left[\frac{K^{2}}{\Delta}+2 \operatorname{irs}\left(2 \omega-\frac{K}{\Delta}\right)+2 m a \omega-a^{2} \omega^{2}-A\right] R=0,
\end{array}
$$

where $s$ is the spin-weight of the fields, $\omega$ is a frequency of the fields, and $K(r)=\left(r^{2}+a^{2}\right) \omega-m a$. 
The solution to the angular Teukolsky equation for brane scalar fields in 5D (3.1) is given by a generalized spheroidal function, and the separation constant is determined in terms of the spin-weighted spheroidal eigenvalue ${ }_{s} \lambda_{l}^{m}( \pm i a \omega)$ which reduces to a spheroidal eigenvalue for $s=0$ case $[18,30]$,

$$
A={ }_{s} \lambda_{l}^{m(a)}( \pm i a \omega)-a^{2} \omega^{2}-s(s+1)
$$

where the superscript ${ }^{(a)}$ denotes the eigenvalue for the angular equation (3.1). With this value $A$, all parameters in the radial equation are fixed.

The radial Teukolsky equation for brane scalar fields in 5D (3.2) possesses two regular singularities in the finite region and one irregular singularity of rank 1 at infinity. Thus the equation transforms to the confluent Heun equation which is the second order linear differential equation with the same singularity structure. After changing variables to dimensionless ones, $a \rightarrow a_{*} \equiv a / r_{\mathrm{h}}, \omega \rightarrow \tilde{\omega} \equiv r_{\mathrm{h}} \omega$, and $r \rightarrow \tilde{r} \equiv r / r_{\mathrm{h}}$, the transformation of the dependent variable, $R(\tilde{r})=(\tilde{r}+1)^{-\frac{s}{2}}(\tilde{r}-1)^{-\frac{s}{2}} \tilde{R}(\tilde{r})$, leads to the confluent Heun equation in the Bôcher symmetrical form, so-called the general spheroidal equation [31],

$$
\frac{\mathrm{d}}{\mathrm{d} \tilde{r}}\left(1-\tilde{r}^{2}\right) \frac{\mathrm{d}}{\mathrm{d} \tilde{r}} \tilde{R}(\tilde{r})+\left[\gamma^{2}\left(1-\tilde{r}^{2}\right)-2 i \gamma \beta \tilde{r}+\lambda-\frac{\mu^{2}+\sigma^{2}+2 \mu \sigma \tilde{r}}{1-\tilde{r}^{2}}\right] \tilde{R}(\tilde{r})=0,
$$

where we find a set of parameters,

$$
\left\{\begin{array}{l}
\gamma=\tilde{\omega} \\
\mu=i\left[\left(a_{*}^{2}+1\right) \tilde{\omega}-m a_{*}\right] \\
\lambda_{\nu}^{\mu}(\gamma)=A-\left(a_{*}^{2}+2\right) \tilde{\omega}^{2}+s(s+1) \\
\beta=\sigma=s
\end{array} .\right.
$$

With this set of parameters we can write down the solution in terms of the confluent Heun functions with the general complex parameters, which has not been studied much. In the case of the scalar fields $(s=0)$, the parameters $\sigma$ and $\beta$ become zero, and the equation (3.4) reduces to the spheroidal equation with general parameters developed by Meixner [32, 33] and explained well with calculation methods in ref. [34]. Therefore, we focus on the $s=0$ case in this paper, in which the Teukolsky equation becomes

$$
\begin{array}{r}
\frac{1}{\sin \theta} \frac{\mathrm{d}}{\mathrm{d} \theta}\left(\sin \theta \frac{\mathrm{d}}{\mathrm{d} \theta} S(\theta)\right)+\left[(a \omega \cos \theta)^{2}-(m \csc \theta)^{2}+A\right] S(\theta)=0, \\
\frac{\mathrm{d}}{\mathrm{d} r}\left(\Delta \frac{\mathrm{d}}{\mathrm{d} r} R(r)\right)+\left[\frac{K^{2}}{\Delta}+2 m a \omega-a^{2} \omega^{2}-A\right] R(r)=0
\end{array}
$$

as we discussed its systematic derivation with uniqueness in section 2 .

In the spheroidal equation, ${ }^{1}$ corresponding to the scalar case, three parameters $\mu$, $\nu$, and $\gamma$ are called the order, degree, and size parameters, respectively. For the real (purely imaginary) size parameter $\gamma$, the equation is called the prolate (oblate) spheroidal equation. A constant $\lambda_{\nu}^{\mu}(\gamma)$ in (3.5) is called the spheroidal eigenvalue and is determined

\footnotetext{
${ }^{1}$ For the spheroidal equation and the related mathematical properties, refer to appendix B.
} 
as the minimal solution [34]. In our case, the eigenvalue $\lambda$ is fixed by the relation with the angular Teukolsky equation (3.5) and so does $\nu$. The parameter domain is given by

$$
\mu, \nu \in \mathbb{C}, \quad \gamma \in \mathbb{R}^{+}, \quad \tilde{r}>1 .
$$

Therefore the solutions for the Teukolsky equation for brane scalar fields in 5D are given by the radial prolate spheroidal functions $S_{\nu}^{\mu}(\tilde{r} ; \gamma)(i=1,2)$ with complex parameters, where the radial in the radial spheroidal function indicates the domain $\tilde{r}>1$ of the spheroidal function, not the radial Teukolsky equation. Since the radial spheroidal function $S_{\nu}^{\mu}(\tilde{r} ; \gamma)$ involves branch cuts in the complex $\tilde{r}$-plane along the semi-infinite line starting at the point $\tilde{r}=0$ and passing through $\tilde{r}=-1 / \gamma$ for non-integer $\nu$, and on the interval $(-1,1)$ for noninteger $\mu / 2$ [34], the branch cut does not lie on the real axis $\tilde{r}>1$ of our consideration.

Since the Wronskian of the radial spheroidal function is proportional to $\left[\tilde{\omega}\left(\tilde{r}^{2}-1\right)\right]^{-1}$ for all parameter values $[32,34]$, the full solution and its eigenvalues of the generalized Teukolsky equation for the brane scalar fields in 5D are given by,

$$
\begin{aligned}
\text { solution : } & R(\tilde{r}) & =C_{1} S_{\nu}^{\mu(1)}(\tilde{r} ; \tilde{\omega})+C_{2} S_{\nu}^{\mu(2)}(\tilde{r} ; \tilde{\omega}), \\
\text { eigenvalues : } & \lambda_{\nu}^{\mu}(\tilde{\omega}) & =\lambda_{l}^{m(a)}\left(i a_{*} \tilde{\omega}\right)-2\left(a_{*}^{2}+1\right) \tilde{\omega}^{2},
\end{aligned}
$$

where $C_{1}$ and $C_{2}$ are constants, and $\mu=i\left[\left(a_{*}^{2}+1\right) \tilde{\omega}-m a_{*}\right]$. Since the spheroidal eigenvalue has the symmetry property,

$$
\lambda_{\nu}^{\mu}(\tilde{\omega})=\lambda_{-\nu-1}^{\mu}(\tilde{\omega})=\lambda_{\nu}^{-\mu}(\tilde{\omega})
$$

we choose one of the three parameter sets, $(\mu, \nu),(\mu,-\nu-1)$, and $(-\mu, \nu)$, and then keep the calculation. The linearly independent solutions $S_{\nu}^{\mu(i)}(\tilde{r} ; \tilde{\omega})$ can be expanded in terms of the spherical Bessel functions, $j, y$,

$$
S_{\nu}^{\mu}{ }^{(i)}(\tilde{r} ; \tilde{\omega})=\frac{\left(1-1 / \tilde{r}^{2}\right)^{\mu / 2}}{A_{\nu}^{-\mu}(\tilde{\omega})} \sum_{k=-\infty}^{\infty} a_{\nu, 2 k}^{-\mu}(\tilde{\omega}) f_{\nu+2 k}(\tilde{\omega} \tilde{r}),
$$

where $i=1,2$, and $f=j, y$, respectively, and the normalization factor $A_{\nu}^{\mu}(\tilde{\omega})$ is given by

$$
A_{\nu}^{\mu}(\tilde{\omega})=\sum_{k=-\infty}^{\infty}(-1)^{k} a_{\nu, 2 k}^{\mu}(\tilde{\omega}) .
$$

As $\tilde{\omega} \tilde{r} \rightarrow \infty$, the factor (3.10) is chosen so that the radial functions have the following asymptotic behaviours [34]:

$$
S_{\nu}^{\mu(1)}(\tilde{r} ; \tilde{\omega}) \longrightarrow j_{\nu}(\tilde{\omega} \tilde{r}), \quad S_{\nu}^{\mu(2)}(\tilde{r} ; \tilde{\omega}) \longrightarrow y_{\nu}(\tilde{\omega} \tilde{r}) .
$$

In the full solution, the series coefficients $a_{\nu, 2 k}^{\mu}(\tilde{r} ; \tilde{\omega})$ satisfy the three-term recurrence relation, and the solutions to the recurrence relations are given as the minimal solution with which the ratio of the coefficients converges to zero, $a_{\nu, 2 k \pm 2}^{\mu}(\tilde{\omega}) / a_{\nu, 2 k}^{\mu}(\tilde{\omega}) \rightarrow 0$, as $k \rightarrow \pm \infty$. This minimal condition is satisfied for the spheroidal eigenvalues $\lambda_{\nu}^{\mu}(\tilde{\omega})$, and the series expansion in the definition absolutely converges for all $\tilde{\omega}$ and $\tilde{r}$. 


\section{Boundary condition near the horizon}

Near the horizon of a black hole, the coefficient of the wave solution corresponding to outgoing wave packet should vanish due to its attractive nature and thus this is called ingoing boundary condition. The asymptotic near-horizon and far-field solutions for the generalized Teukolsky equation (3.2) were obtained in the previous research [18]

\begin{tabular}{|cc|cc|}
\hline \multicolumn{2}{|c|}{$r \rightarrow \infty$} & \multicolumn{2}{|c|}{$r \rightarrow r_{\mathrm{h}}$} \\
\hline ingoing & outgoing & ingoing & outgoing \\
\hline$\frac{1}{r} e^{-i \omega r_{*}}$ & $\frac{1}{r^{2 s+1}} e^{i \omega r_{*}}$ & $e^{-i k r_{*}} \Delta^{-s}$ & $e^{i k r_{*}}$ \\
$\rightarrow \frac{1}{r} e^{-i \omega r}$ & $\rightarrow \frac{1}{r} e^{i \omega r}$ & $\rightarrow(\tilde{r}-1)^{-\frac{\mu}{2}}$ & $\rightarrow(\tilde{r}-1)^{\frac{\mu}{2}}$ \\
\hline
\end{tabular}

where the tortoise coordinate $r_{*}$ was used, $k=\omega-m a /\left(r_{\mathrm{h}}^{2}+a^{2}\right)$, and $d r_{*} / d r=\left(r^{2}+a^{2}\right) / \Delta(r)$. By definition, the coordinate $r_{*}$ approaches $r, r_{*} \rightarrow r$ for $r \rightarrow \infty$. In the last row of the table, the asymptotic solutions are written in the Boyer-Lindquist coordinates. To identify the ingoing boundary condition, we need to find two linear combinations of the two solutions $S_{\nu}^{\mu(i)}(\tilde{r} ; \tilde{\omega})(i=1,2)$ in order that the two resultant functions become either ingoing or outgoing solution in the near horizon limit, respectively. Overall factors of the chosen solutions $S_{\nu}^{\mu(i)}(\tilde{r} ; \tilde{\omega})(i=1,2)$ in (3.10) match the outgoing asymptotic solution in the near-horizon limit in the table, however there remain nontrivial series terms in the solution to be examined. Therefore, a different linear combination matching the ingoing asymptotic solution should be investigated in what follows by using the properties of the spheroidal functions, which are nontrivial.

The spheroidal functions have joining relations between the second kind angular spheroidal function of type II, $\mathrm{Qs}_{\nu}^{\mu}(\tilde{r} ; \tilde{\omega})$, which is expanded in terms of the associated Legendre functions of type II, $\mathfrak{Q}_{\nu}^{\mu}(\tilde{r} ; \tilde{\omega})$, and the radial spheroidal function, $S_{\nu}^{\mu(1)}(\tilde{r} ; \tilde{\omega})$ as, ${ }^{2}$

$$
\begin{aligned}
& S_{\nu}^{\mu(1)}(\tilde{r} ; \tilde{\omega})=K_{\nu}^{\mu}(\tilde{\omega}) \frac{\sin ((\mu-\nu) \pi)}{\pi} \frac{\mathrm{e}^{-i(\mu+\nu) \pi}\left(1-1 / \tilde{r}^{2}\right)^{\mu / 2}(\tilde{\omega} \tilde{r})^{\nu}}{\tilde{\omega}^{\nu} \tilde{r}^{\nu-\mu}(\tilde{r}-1)^{\mu / 2}(\tilde{r}+1)^{\mu / 2}} \mathrm{Qs}_{-\nu-1}^{\mu}(\tilde{r} ; \tilde{\omega}), \\
& S_{\nu}^{\mu(2)}(\tilde{r} ; \tilde{\omega})=-\sec (\nu \pi)\left[S_{-\nu-1}^{\mu(1)}(\tilde{r} ; \tilde{\omega})+\sin (\nu \pi) S_{\nu}^{\mu(1)}(\tilde{r} ; \tilde{\omega})\right]
\end{aligned}
$$

where the factor $K_{\nu}^{\mu}(\tilde{\omega})$ in front of the first function is called the spheroidal joining factor and its exact form is given by, ${ }^{3}$

$$
K_{\nu}^{\mu}(\tilde{\omega})=\mathrm{e}^{i \nu \pi} 2^{-2 \nu-1} \Gamma(\nu-\mu+1) \frac{\tilde{\omega}^{\nu}}{A_{\nu}^{-\mu}(\tilde{\omega})} \frac{\sum_{k=0}^{\infty} \frac{(-1)^{k} a_{\nu,-2 k}^{-\mu}(\tilde{\omega})}{\Gamma\left(-k+\nu+\frac{3}{2}\right) k !}}{\sum_{k=0}^{\infty} \frac{(-1)^{k} a_{\nu, 2 k}^{\mu}(\tilde{\omega})}{\Gamma\left(-k-\nu+\frac{1}{2}\right) k !}} .
$$

\footnotetext{
${ }^{2}$ In the reference [34], the relation for $S_{\nu}^{\mu(2)}$ is given with wrong signs.

${ }^{3}$ In the reference [34], this relation is given with missing $(-1)^{k}$ factor in the denominator.
} 
The symmetry relations of the aforementioned the second kind angular spheroidal function of type II, $\mathrm{Qs}_{\nu}^{\mu}(\tilde{r} ; \tilde{\omega})$, and those of the first kind angular spheroidal function of type II, $\operatorname{Ps}_{\nu}^{\mu}(\tilde{r} ; \tilde{\omega})$, are given as

$$
\begin{aligned}
\mathrm{Qs}_{-\nu-1}^{\mu}(\tilde{r} ; \tilde{\omega}) & =\csc ((\mu-\nu) \pi)\left[\pi e^{i \mu \pi} \cos (\nu \pi) \operatorname{Ps}_{\nu}^{\mu}(\tilde{r} ; \tilde{\omega})-\sin ((\mu+\nu) \pi) \mathrm{Qs}_{\nu}^{\mu}(\tilde{r} ; \tilde{\omega})\right] \\
\operatorname{Ps}_{\nu}^{-\mu}(\tilde{r} ; \tilde{\omega}) & =\frac{\Gamma(\nu-\mu+1)}{\Gamma(\nu+\mu+1)}\left[\operatorname{Ps}_{\nu}^{\mu}(\tilde{r} ; \tilde{\omega})-\frac{2}{\pi} e^{-i \mu \pi} \sin (\mu \pi) \operatorname{Qs}_{\nu}^{\mu}(\tilde{r} ; \tilde{\omega})\right]
\end{aligned}
$$

where $\operatorname{Ps}_{\nu}^{\mu}(\tilde{r} ; \tilde{\omega})$ is expanded in terms of the associated Legendre functions of type II, $\mathfrak{P}_{\nu}^{\mu}(\tilde{r} ; \tilde{\omega})$. With the help of (4.2) and (4.3), we write down the full solutions as a linear combination of the first kind angular spheroidal functions of type II with different parameters, $\operatorname{Ps}_{\nu}^{\mu}(\tilde{r} ; \tilde{\omega})$ and $\operatorname{Ps}_{\nu}^{-\mu}(\tilde{r} ; \tilde{\omega})$ as

$$
R(\tilde{r})=C_{+} \operatorname{Ps}_{\nu}^{\mu}(\tilde{r} ; \tilde{\omega})+C_{-} \operatorname{Ps}_{\nu}^{-\mu}(\tilde{r} ; \tilde{\omega}),
$$

where the coefficients $C_{+}$and $C_{-}$are given by,

$$
\begin{aligned}
C_{+}= & \frac{1}{2}(1-i \tan (\nu \pi)) \frac{1}{\sin (\mu \pi)} \times \\
& \times\left[\sin ((\mu-\nu) \pi)\left(C_{1} \cos (\nu \pi)-C_{2} \sin (\nu \pi)\right) K_{\nu}^{\mu}(\tilde{\omega})-C_{2} e^{2 i \nu \pi} \sin ((\mu+\nu) \pi) K_{-\nu-1}^{\mu}(\tilde{\omega})\right], \\
C_{-}= & \frac{1}{2}(1-i \tan (\nu \pi)) \frac{\sin ((\mu+\nu) \pi)}{\sin (\mu \pi)} \frac{\Gamma(\nu+\mu+1)}{\Gamma(\nu-\mu+1)} \times \\
& \times\left[\left(C_{1} \cos (\nu \pi)-C_{2} \sin (\nu \pi)\right) K_{\nu}^{\mu}(\tilde{\omega})+C_{2} e^{2 i \nu \pi} K_{-\nu-1}^{\mu}(\tilde{\omega})\right] .
\end{aligned}
$$

With this new form of the full solution, eq. (4.4), we can directly read the ingoing or outgoing solutions as follows. By definition, the two solutions $\operatorname{Ps}_{\nu}^{ \pm \mu}(\tilde{r} ; \tilde{\omega})$ are written in terms of the first kind associated Legendre functions of type I, $\mathfrak{P}$ 's,

$$
\begin{aligned}
\operatorname{Ps}_{\nu}^{ \pm \mu}(\tilde{r} ; \tilde{\omega})= & \sum_{k=-\infty}^{\infty}(-1)^{k} a_{\nu, 2 k}^{ \pm \mu}(\tilde{\omega}) \mathfrak{P}_{\nu+2 k}^{ \pm \mu}(\tilde{r}) \\
= & \frac{1}{\Gamma(1 \mp \mu)} \frac{(\tilde{r}+1)^{ \pm \mu / 2}}{(\tilde{r}-1)^{ \pm \mu / 2}} \\
& \times \sum_{k=-\infty}^{\infty}(-1)^{k} a_{\nu, 2 k}^{ \pm \mu}(\tilde{\omega})_{2} F_{1}\left(-\nu-2 k, \nu+2 k+1 ; 1 \mp \mu ; \frac{1-\tilde{r}}{2}\right),
\end{aligned}
$$

where ${ }_{2} F_{1}(a, b ; c ; z)$ is Gaussian hypergeometric function. Notice that, in the near horizon limit of $\tilde{r} \rightarrow 1$, the series part of (4.6) converges to the normalization factors $A_{\nu}^{ \pm \mu}(\tilde{\omega})$ by its definition eq. (3.11). Then, the overall factor in front of the series determines the leading behavior of the only remaining dominant terms read the ingoing and outgoing asymptotic solutions. Subsequently the coefficients $C_{+}$and $C_{-}$in (4.4), specifically (4.5), are identified as those of ingoing and outgoing solutions near the horizon, respectively. By setting the outgoing wave coefficient zero, $C_{-}=0$, we find the ingoing boundary condition,

$$
\frac{C_{1}}{C_{2}}=\frac{1}{\cos (\pi \nu)}\left[\sin (\pi \nu)-e^{2 i \nu \pi} \frac{K_{-\nu-1}^{\mu}(\tilde{\omega})}{K_{\nu}^{\mu}(\tilde{\omega})}\right]
$$


According to the fixation of the ingoing boundary condition, the ratio of the two spheroidal joining factors, $K_{-\nu-1}^{\mu}(\tilde{\omega}) / K_{\nu}^{\mu}(\tilde{\omega})$, determines the ratio of two coefficients, $C_{1} / C_{2}$, in the full solution (3.8) up to an overall constant. Note that the ratio $C_{1} / C_{2}$ in (4.7) will be applied only for integral $\nu$ 's in the low frequency limit, where the denominator $\cos (\pi \nu)$ becomes \pm 1 . Now that we have the ingoing boundary condition of the Teukolsky equation for brane scalar fields in 5D, we are ready to obtain its greybody factors.

\section{Greybody factors for scalar fields}

The greybody factor for black holes, $\Gamma$, is the correction factor to the Hawking radiation which is described as a black body radiation. This correction factor is calculated from the absorption probability of the incoming wave. For brane scalar fields, the greybody factor $\Gamma$ is given in terms of the ratio between the coefficients of ingoing and outgoing far field solutions [37],

$$
\Gamma=1-\left|\frac{C_{\text {out }}^{\infty}}{C_{\text {in }}^{\infty}}\right|^{2}
$$

The coefficients of the far field solutions, $C_{\text {in }}^{\infty}$ and $C_{\text {out }}^{\infty}$, are computed by comparing with the asymptotic behavior of the radial spheroidal function in eq. (3.12),

$$
\begin{aligned}
R(\tilde{r}) & =C_{1} S_{\nu}^{\mu(1)}(\tilde{r} ; \tilde{\omega})+C_{2} S_{\nu}^{\mu(2)}(\tilde{r} ; \tilde{\omega}) \\
& \rightarrow C_{1} j_{\nu}(\tilde{\omega} \tilde{r})+C_{2} y_{\nu}(\tilde{\omega} \tilde{r}) \\
& \equiv C_{\text {in }}^{\infty}\left(\frac{1}{r} e^{-i \omega r}\right)+C_{\text {out }}^{\infty}\left(\frac{1}{r} e^{i \omega r}\right),
\end{aligned}
$$

where in the last line $\omega r$ was written as the same value $\tilde{\omega} \tilde{r}=\omega r$. Thus, $C_{\text {in }}^{\infty}$ and $C_{\text {out }}^{\infty}$ are expressed in terms of the coefficients of the spheroidal functions, $C_{1}$ and $C_{2}$,

$$
C_{\mathrm{in}}^{\infty}=\frac{C_{1}+i C_{2}}{2 \tilde{\omega}} e^{i \frac{(\nu+1)}{2} \pi}, \quad C_{\text {out }}^{\infty}=\frac{C_{1}-i C_{2}}{2 \tilde{\omega}} e^{-i \frac{(\nu+1)}{2} \pi} .
$$

Substitution of (5.3) into the greybody factor for brane scalar fields (5.1) gives

$$
\Gamma=1-\left|-\frac{1+i\left(C_{1} / C_{2}\right)}{1-i\left(C_{1} / C_{2}\right)} e^{-i(\nu+1) \pi}\right|^{2} .
$$

Since the ratio $C_{1} / C_{2}$ is fixed by the ingoing boundary condition in eq. (4.7), we obtain the greybody factor in terms of the ratio between the spheroidal joining factors,

$$
\Gamma=1-\left|\frac{i e^{i \nu \pi}+e^{2 i \nu \pi}\left(K_{-\nu-1}^{\mu}(\tilde{\omega}) / K_{\nu}^{\mu}(\tilde{\omega})\right)}{-i+e^{3 i \nu \pi}\left(K_{-\nu-1}^{\mu}(\tilde{\omega}) / K_{\nu}^{\mu}(\tilde{\omega})\right)}\right|^{2} .
$$

As a consistency check, low frequency limit is taken for the obtained solutions and greybody factors, and it is compared to the known approximate results in [18], whose near-horizon asymptotic solution for brane scalar fields is

$$
\begin{aligned}
R_{\mathrm{NH}}= & C_{1}^{\prime}\left(\frac{\tilde{r}-1}{2}\right)^{-\frac{\mu}{2}}\left(\frac{\tilde{r}+1}{2}\right)^{\frac{\mu}{2}}{ }_{2} F_{1}\left(-l, l+1,1-\mu, \frac{1-\tilde{r}}{2}\right) \\
& +C_{2}^{\prime}\left(\frac{\tilde{r}-1}{2}\right)^{\frac{\mu}{2}}\left(\frac{\tilde{r}+1}{2}\right)^{\frac{\mu}{2}}{ }_{2} F_{1}\left(-l+\mu, l+\mu+1,1+\mu, \frac{1-\tilde{r}}{2}\right) .
\end{aligned}
$$


First, in the limit of low energy $\tilde{\omega} \rightarrow 0$, the ratio of two coefficients in the definition of the radial spheroidal function in eq. (3.10) behaves [34]

$$
\frac{a_{\nu, \pm k}^{\mu}(\tilde{\omega})}{a_{\nu, 0}^{\mu}(\tilde{\omega})} \sim \mathcal{O}\left(\tilde{\omega}^{2 k}\right), \quad(k=0,1,2, \cdots)
$$

and thus the $a_{\nu, 0}^{\mu}(\tilde{\omega})$ term is dominant. Second, the $\nu$ of the solution $S_{\nu}^{\mu(1)}$ or $S_{\nu}^{\mu(2)}$ in (3.8) becomes the integer parameter $l$ of angular eigenvalues (3.3) by the asymptotic property of the spheroidal eigenvalue [34]. In this limit, with the help of a property of the hypergeometric function, ${ }_{2} F_{1}(a, b, c, z)=z^{1-c}{ }_{2} F_{1}(1+a-c, 1+b-c, 2-c, z)$ for nonintegral $c$ and a symmetry of the first kind of the angular spheroidal function of type II, $\operatorname{Ps}_{\nu}^{\mu}(\tilde{r} ; \tilde{\omega})=\operatorname{Ps}_{-\nu-1}^{\mu}(\tilde{r} ; \tilde{\omega})$, the full solution in eq. (4.4) turns out to be the same as the approximate solution in eq. (5.6) up to a normalization constant.

In the low frequency limit, the greybody factor is simplified as

$$
\Gamma=1-\left|\frac{1+i\left(C_{1} / C_{2}\right)}{1-i\left(C_{1} / C_{2}\right)}\right|^{2}
$$

where the ingoing boundary condition is simplified

$$
\frac{C_{1}}{C_{2}}=(-1)^{l+1} \frac{K_{-l-1}^{\mu}(\tilde{\omega})}{K_{l}^{\mu}(\tilde{\omega})} .
$$

Due to eq. (5.7), the spheroidal joining factor in eq. (4.2) approaches its asymptotic form

$$
K_{\nu}^{\mu}(\tilde{\omega}) \rightarrow e^{i l \pi} 2^{-2 l-1} \Gamma(l-\mu+1) \frac{\tilde{\omega}^{l}}{A_{l}^{-\mu}(\tilde{\omega})} \frac{a_{l, 0}^{-\mu}(\tilde{\omega}) \Gamma\left(-l+\frac{1}{2}\right)}{a_{l, 0}^{\mu}(\tilde{\omega}) \Gamma\left(l+\frac{3}{2}\right)} .
$$

Using the symmetry relations of the normalizing constant, $A_{\nu}^{\mu}(\gamma)=A_{-\nu-1}^{\mu}(\gamma)$, and spheroidal expansion coefficients, $a_{l, 0}^{\mu}(\gamma)=a_{l, 0}^{-\mu}(\gamma)$, the ingoing boundary condition (5.9) becomes

$$
\frac{C_{1}}{C_{2}}=(-1)^{l+1} \frac{4^{-2 l-1} \tilde{\omega}^{2 l+1} \Gamma\left(\frac{1}{2}-l\right)^{2} \Gamma(l-\mu+1)}{\Gamma\left(\frac{3}{2}+l\right)^{2} \Gamma(-l-\mu)},
$$

where (5.9) is also used. Straightforward computation of the gamma function in eq. (5.11) leads exactly to the same equation in [18]. ${ }^{4}$ In figure 1 , the exact solutions and approximate solutions in the low frequency limit are compared for the simplest mode, $(l, m)=(0,0)$. The rotation parameter $a_{*}$ varies from zero to the maximum possible value $a_{* \max }=1.5$.

In figure 2, the greybody factor curves of the exact solutions are also compared to the numerical results [19]. We plot these two results for the various $l=m$ modes with the rotation parameter, $a_{*}=a_{* \max }$, whose graphs show overlaps.

\footnotetext{
${ }^{4}$ In the ref. [18], the greybody factor was written in terms of $C \equiv-i\left(C_{1} / C_{2}\right)$.
} 


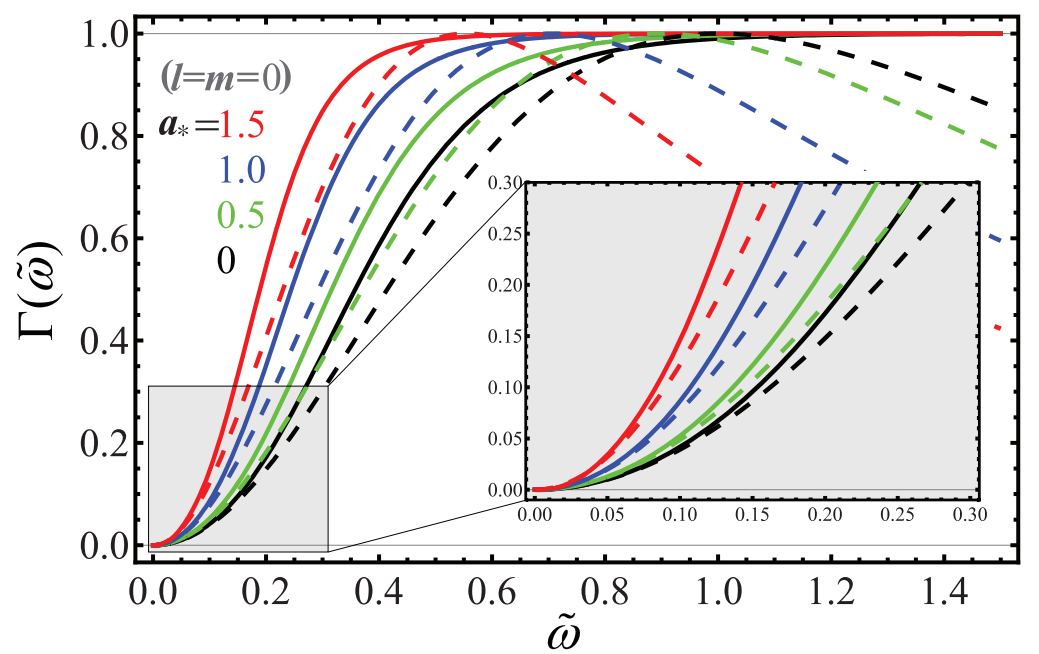

Figure 1. Greybody factor curves of $l=m=0$ modes are compared for various rotation parameters $a_{*}$ : solid lines denote the graphes of exact solutions and dashed lines those of approximate solutions in the low frequency limit.

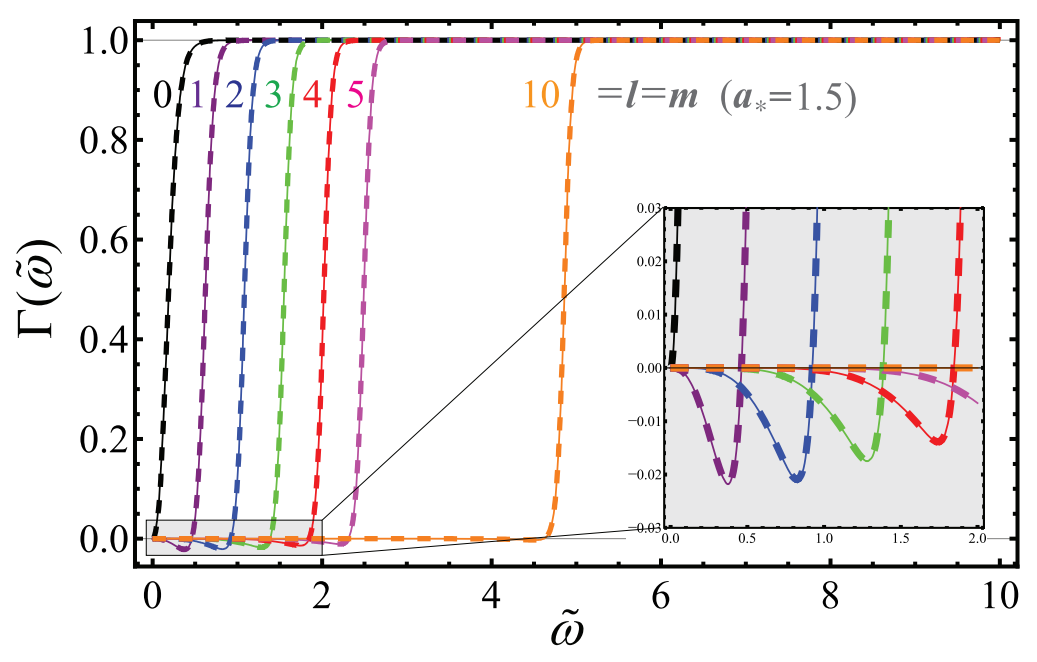

Figure 2. Greybody factor curves of exact solutions (thin solid lines) and those of numerical results (dashed lines) are compared for $l=m$ modes with $a_{*}=a_{* \max }$.

\section{Summary and discussions}

In low scale gravity scenarios, higher dimensional rotating black holes are copiously produced in high energy collisions, which may be within the reach of the high energy LHC or future circular collider at CERN or China with $\sqrt{s}=100 \mathrm{TeV}$. The detection of Hawking radiation from a higher dimensional rotating black hole may be the most interesting way of proving not only the large or warped extra dimensions, ${ }^{5}$ but also the quantum phenomenon of gravity [35].

\footnotetext{
${ }^{5}$ In [36], black holes from the compact hyperbolic extra dimensions are discussed.
} 
To support the experimental searches of such signal and theoretical interest by its own, there have been significant theoretical studies on the Hawking radiation for higher dimensional rotating black holes in both the analytical and numerical ways. Before this paper, the analytic studies have been successful only in the specific regimes, e.g., lowfrequency regime or high-frequency regime. In the present work, we are successful in finding the full solution of the generalized Teukolsky equation without assuming any limitation in the frequency domain for the brane scalar modes in 5D black hole background of MyersPerry solution.

The obtained exact result enables us to describe the Hawking radiation in the entire frequency regime and to analyze its characters in detail. Since the detailed study of greybody factors allow us to have a better understanding of quantum nature of black holes, the study with various approaches will give us some insight into the fundamental properties of black holes. Therefore the exact form of the greybody factor of scalar perturbations for the 5D black holes may provide a good approach for the extra dimension search and development of quantum gravity.

Finally, we comment on the Teukolsky equation for the higher spin fields with $s=$ $1 / 2,1,2 / 3$, and 2 . We have shown that those high spin states are governed by the confluent Heun equation with complex parameters, which still lack of understanding its basic mathematical properties, yet. Intriguingly, the equation has the same mathematical structure with that of the four-dimensional Kerr black hole [38-40]. In addition, Kerr-Newman-de Sitter black holes also lead to Heun-type solutions for a massive, electrically charged scalar particle [41]. Thus, finding analytic solutions in one side would provide the greybody factor on the other side, which is definitely worth more attention for the future.

\section{Acknowledgments}

Y.-H. Hyun, Y. Kim, and S.C. Park are supported by Basic Science Research Program through the National Research Foundation of Korea (NRF) funded by the Ministry of Science, ICT and Future Planning (Grant No. NRF-2018R1D1A1B07049514, NRF2016R1D1A1B03931090, and NRF-2016R1A2B2016112 \& NRF-2018R1A4A1025334, respectively). Y.-H. Hyun is also supported by the Korea Institute of Science and Technology Information (K-18-L12-C08-S01).

\section{A Myers-Perry black holes}

The $d$-dimensional flat space metric of $n=[(d-1) / 2](n \geq 2)$ can take [29],

$$
\begin{aligned}
d s^{2} & =-\mathrm{d} t^{2}+\sum_{i=1}^{n}\left(\mathrm{~d} x_{i}^{2}+\mathrm{d} y_{i}^{2}\right)+\left.\mathrm{d} z^{2}\right|_{d_{\text {even }}} \\
& =-\mathrm{d} t^{2}+\mathrm{d} r^{2}+r^{2} \sum_{i=1}^{n}\left(\mathrm{~d} \mu_{i}^{2}+\mu_{i}^{2} \mathrm{~d} \phi_{i}^{2}\right)+\left.r^{2} \mathrm{~d} \alpha^{2}\right|_{d_{\text {even }}},
\end{aligned}
$$


where the paired spatial Cartesian coordinates $\left(x_{i}, y_{i}\right)$ in $n$ orthogonal planes is expressed in terms of the polar coordinates $\left(\mu_{i}, \phi_{i}\right)$ whose direction cosines are

$$
\begin{aligned}
& x_{i}=r \mu_{i} \cos \phi_{i}, \quad y_{i}=r \mu_{i} \sin \phi_{i}, \quad z=r \alpha \\
& r^{2}=\sum_{i=1}^{n}\left(x_{i}^{2}+y_{i}^{2}\right), \quad \sum_{i=1}^{n} \mu_{i}^{2}+\left.\alpha^{2}\right|_{d_{\mathrm{even}}}=1 .
\end{aligned}
$$

With this setup, the solutions of arbitrary rotation in every independent rotation plane are given by

$$
\begin{aligned}
d s^{2}= & -\mathrm{d} t^{2}+\frac{\mu r \cdot\left(\left.r\right|_{d_{\text {even }}}\right)}{\Pi F}\left(\mathrm{~d} t+\sum_{i=1}^{n} a_{i} \mu_{i}^{2} \mathrm{~d} \phi_{i}\right)^{2}+\frac{\Pi F}{\Pi-\mu r \cdot\left(\left.r\right|_{d_{\text {even }}}\right)} \mathrm{d} r^{2} \\
& +\left.r^{2} \mathrm{~d} \alpha^{2}\right|_{d_{\text {even }}}+\sum_{i=1}^{n}\left(r^{2}+a_{i}^{2}\right)\left(\mathrm{d} \mu_{i}^{2}+\mu_{i}^{2} \mathrm{~d} \phi_{i}^{2}\right),
\end{aligned}
$$

where the number of independent rotation planes is $n=\left[\frac{d-1}{2}\right]$ and

$$
\mu_{i}^{2}+\alpha^{2}=1, \quad F=1-\sum_{i=1}^{n} \frac{a_{i}^{2} \mu_{i}^{2}}{r^{2}+a_{i}^{2}}, \quad \Pi=\prod_{i=1}^{n}\left(r^{2}+a_{i}^{2}\right) .
$$

In the obtained metric, $\left(\left.r\right|_{d_{\text {even }}},\left.d \alpha^{2}\right|_{d_{\text {even }}}\right)$ reduce to $(1,0)$ for $d$ odd dimensions. By examining the asymptotic structure of these metrics, the $(n+1)$ free parameters, $\mu$ and $a_{i}$, determine respectively the mass and angular momenta carried by the black hole,

$$
M=\frac{(d-2) \Omega_{d-2}}{16 \pi G} \mu, \quad J^{y_{i} x_{i}}=\frac{\Omega_{d-2}}{8 \pi G} \mu a_{i}=\frac{2}{d-2} M a_{i} .
$$

\section{B Spheroidal equation}

The spheroidal equation takes the form,

$$
\frac{\mathrm{d}}{\mathrm{d} z}\left(1-z^{2}\right) \frac{\mathrm{d}}{\mathrm{d} z} w(z)+\left[\gamma^{2}\left(1-z^{2}\right)+\lambda-\frac{\mu^{2}}{1-z^{2}}\right] w(z)=0,
$$

where the parameters $\mu, \nu$, and $\gamma$ are called the order, degree, and size parameters, respectively. According to the parameters, the spheroidal equation (B.1) is classified by four equations as

\begin{tabular}{|c|c|}
\hline parameter & name of spheroidal equation \\
\hline$|z|<1, \gamma \in \mathbb{R}$ & angular prolate spheroidal equation \\
\hline$|z|<1, \gamma \in i \mathbb{R}$ & angular oblate spheroidal equation \\
\hline$|z|>1, \gamma \in \mathbb{R}$ & radial prolate spheroidal equation \\
\hline$|z|>1, \gamma \in i \mathbb{R}$ & radial oblate spheroidal equation \\
\hline
\end{tabular}


For the real (purely imaginary) size parameter $\gamma$, the equation is called the prolate (oblate) spheroidal equation. The constant $\lambda_{\nu}^{\mu}(\gamma)$ is called spheroidal eigenvalue and is determined as the minimal solution [34]. When $\gamma=0$ and $|z|<1$, the equation (B.1) reduces to Legendre differential equation. When $f(z)=\left(1-1 / z^{2}\right)^{\mu / 2} g(\gamma z), \gamma \rightarrow 0$, and $|z|>1$, it reduces to the spherical Bessel equation. The solutions to this equation for the separated domain is defined as,

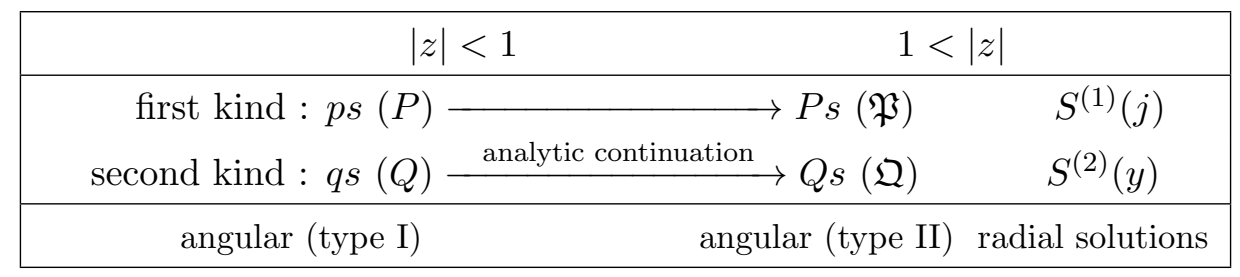

where the expansion functions in the parenthesis are $P, Q, \mathfrak{P}, \mathfrak{Q}$ (associated Legendre functions) and $j, y$ (spherical Bessel functions).

The angular and radial solutions in the table are expanded as

$$
\begin{aligned}
& F_{\nu}^{\mu}(z ; \gamma)=\sum_{k=-\infty}^{\infty}(-1)^{k} a_{\nu, 2 k}^{\mu}(\gamma) f_{\nu+2 k}^{\mu}(z), \quad(F=p s, q s, P s, Q s, f=P, Q, \mathfrak{P}, \mathfrak{Q}) \\
& S_{\nu}^{\mu(i)}(z ; \gamma)=\frac{\left(1-1 / z^{2}\right)^{\mu / 2}}{A_{\nu}^{-\mu}(\gamma)} \sum_{k=-\infty}^{\infty} a_{\nu, 2 k}^{-\mu} f_{\nu+2 k}(\gamma z), \quad(f=j, y)
\end{aligned}
$$

where the coefficient $a_{\nu, 2 k}^{\mu}$ and the normalization factor $A_{\nu}^{\mu}(\gamma)$ satisfy $^{6}$

$$
\begin{aligned}
& A_{\nu, 2 k}^{\mu}(\gamma) a_{\nu, 2 k-2}^{\mu}(\gamma)+\left(B_{\nu, 2 k}^{\mu}(\gamma)-\lambda\right) a_{\nu, 2 k}^{\mu}(\gamma)+C_{\nu, 2 k}^{\mu}(\gamma) a_{\nu, 2 k+2}^{\mu}(\gamma)=0 \\
& \left\{\begin{array}{l}
A_{\nu, 2 k}^{\mu}(\gamma)=-\gamma^{2} \frac{(\nu-\mu+2 k-1)(\nu-\mu+2 k)}{(2 \nu+4 k-3)(2 \nu+4 k-1)} \\
B_{\nu, 2 k}^{\mu}(\gamma)=(\nu+2 k)(\nu+2 k+1)-2 \gamma^{2} \frac{(\nu+2 k)(\nu+2 k+1)+\mu^{2}-1}{(2 \nu+4 k-1)(2 \nu+4 k+3)} \\
C_{\nu, 2 k}^{\mu}(\gamma)=-\gamma^{2} \frac{(\nu+\mu+2 k+1)(\nu+\mu+2 k+2)}{(2 \nu+4 k+3)(2 \nu+4 k+5)}
\end{array}\right. \\
& A_{\nu}^{\mu}(\gamma)=\sum_{k=-\infty}^{\infty}(-1)^{k} a_{\nu, 2 k}^{\mu}(\gamma)
\end{aligned}
$$

The series expansions of the angular and radial solutions are convergent only when the coefficients $a_{\nu, 2 k}^{\mu}(\gamma)$ form a minimal solution to the recurrence relation. To be specific, a solution with the property that $a_{\nu, 2 k \pm 2}^{\mu}(\gamma) / a_{\nu, 2 k}^{\mu}(\gamma) \rightarrow 0$ as $k \rightarrow \pm \infty$ converges for all values of $z$. There is a countably infinite set of values of $\lambda$, which correspond to minimal solutions. The spheroidal eigenvalue $\lambda_{\nu}^{\mu}(\gamma)$ is defined as a function of $\mu, \nu$, and $\gamma$ by choosing the minimal $\lambda$ value which reduces to $\nu(\nu+1)$ continuously in the limit of $\gamma \rightarrow 0$ along the line $(0, \gamma)[34]$. The expansion of the radial solution is absolutely convergent for $|z|>1$,

\footnotetext{
${ }^{6}$ Typos in the recurrence relation in the ref. [34] has been corrected in the $a_{\nu, 2 k}^{\mu}$ indices and numerical factors in the denominator of the $B_{\nu, 2 k}^{\mu}$ relation.
} 
and the normalization factor $A_{\nu}^{\mu}(\gamma)$ is chosen so that the radial function shows the following behaviour for $\gamma z \rightarrow \infty$,

$$
S_{\nu}^{\mu(1)} \rightarrow \frac{1}{\gamma z} \sin \left(\gamma z-\frac{\nu \pi}{2}\right), \quad S_{\nu}^{\mu(2)} \rightarrow-\frac{1}{\gamma z} \cos \left(\gamma z-\frac{\nu \pi}{2}\right) .
$$

The functions $S_{\nu}^{\mu(1,2)}(z ; \gamma)$ have branch cuts in the complex $z$-plane. One branch cut lies along the semi-infinite line which begins at the point $z=0$ and passes $z=-1 / \gamma$ for non-integer $\nu$, and the other branch cut does on the interval $(-1,1)$ for non-integer $\mu / 2$.

Open Access. This article is distributed under the terms of the Creative Commons Attribution License (CC-BY 4.0), which permits any use, distribution and reproduction in any medium, provided the original author(s) and source are credited.

\section{References}

[1] N. Arkani-Hamed, S. Dimopoulos and G.R. Dvali, The Hierarchy problem and new dimensions at a millimeter, Phys. Lett. B 429 (1998) 263 [hep-ph/9803315] [InSPIRE].

[2] I. Antoniadis, N. Arkani-Hamed, S. Dimopoulos and G.R. Dvali, New dimensions at a millimeter to a Fermi and superstrings at a TeV, Phys. Lett. B 436 (1998) 257 [hep-ph/9804398] [INSPIRE].

[3] N. Arkani-Hamed, S. Dimopoulos and G.R. Dvali, Phenomenology, astrophysics and cosmology of theories with submillimeter dimensions and TeV scale quantum gravity, Phys. Rev. D 59 (1999) 086004 [hep-ph/9807344] [INSPIRE].

[4] L. Randall and R. Sundrum, A Large mass hierarchy from a small extra dimension, Phys. Rev. Lett. 83 (1999) 3370 [hep-ph/9905221] [INSPIRE].

[5] L. Randall and R. Sundrum, An Alternative to compactification, Phys. Rev. Lett. 83 (1999) 4690 [hep-th/9906064] [INSPIRE].

[6] S. Dimopoulos and G.L. Landsberg, Black holes at the LHC, Phys. Rev. Lett. 87 (2001) 161602 [hep-ph/0106295] [INSPIRE].

[7] S.B. Giddings and S.D. Thomas, High-energy colliders as black hole factories: The End of short distance physics, Phys. Rev. D 65 (2002) 056010 [hep-ph/0106219] [InSPIRE].

[8] L.A. Anchordoqui, J.L. Feng, H. Goldberg and A.D. Shapere, Black holes from cosmic rays: Probes of extra dimensions and new limits on TeV scale gravity, Phys. Rev. D 65 (2002) 124027 [hep-ph/0112247] [INSPIRE].

[9] J.L. Feng and A.D. Shapere, Black hole production by cosmic rays, Phys. Rev. Lett. 88 (2002) 021303 [hep-ph/0109106] [INSPIRE].

[10] A. Ringwald and $\mathrm{H}$. Tu, Collider versus cosmic ray sensitivity to black hole production, Phys. Lett. B 525 (2002) 135 [hep-ph/0111042] [INSPIRE].

[11] Y. Jho and S.C. Park, Constraining New Physics with High Multiplicity: I. Ultra-High Energy Cosmic Rays on air-shower detector arrays, arXiv:1806.03063 [INSPIRE].

[12] M. Cavaglia, Black hole and brane production in TeV gravity: A Review, Int. J. Mod. Phys. A 18 (2003) 1843 [hep-ph/0210296] [INSPIRE]. 
[13] P. Kanti, Black holes in theories with large extra dimensions: A Review, Int. J. Mod. Phys. A 19 (2004) 4899 [hep-ph/0402168] [INSPIRE].

[14] S.C. Park, Black holes and the LHC: A Review, Prog. Part. Nucl. Phys. 67 (2012) 617 [arXiv: 1203.4683] [INSPIRE].

[15] S.W. Hawking, Particle Creation by Black Holes, Commun. Math. Phys. 43 (1975) 199 [Erratum ibid. 46 (1976) 206] [INSPIRE].

[16] R. Emparan, G.T. Horowitz and R.C. Myers, Black holes radiate mainly on the brane, Phys. Rev. Lett. 85 (2000) 499 [hep-th/0003118] [INSPIRE].

[17] S.A. Teukolsky, Rotating black holes - separable wave equations for gravitational and electromagnetic perturbations, Phys. Rev. Lett. 29 (1972) 1114 [INSPIRE].

[18] D. Ida, K.-y. Oda and S.C. Park, Rotating black holes at future colliders: Greybody factors for brane fields, Phys. Rev. D 67 (2003) 064025 [Erratum ibid. D 69 (2004) 049901] [hep-th/0212108] [inSPIRE].

[19] D. Ida, K.-y. Oda and S.C. Park, Rotating black holes at future colliders. II. Anisotropic scalar field emission, Phys. Rev. D 71 (2005) 124039 [hep-th/0503052] [INSPIRE].

[20] D. Ida, K.-y. Oda and S.C. Park, Rotating black holes at future colliders. III. Determination of black hole evolution, Phys. Rev. D 73 (2006) 124022 [hep-th/0602188] [INSPIRE].

[21] S. Creek, O. Efthimiou, P. Kanti and K. Tamvakis, Greybody factors for brane scalar fields in a rotating black-hole background, Phys. Rev. D 75 (2007) 084043 [hep-th/0701288] [INSPIRE].

[22] S. Creek, O. Efthimiou, P. Kanti and K. Tamvakis, Greybody factors in a rotating black-hole background. II. Fermions and gauge bosons, Phys. Rev. D 76 (2007) 104013 [arXiv: 0707.1768] [INSPIRE].

[23] R. Jorge, E.S. de Oliveira and J.V. Rocha, Greybody factors for rotating black holes in higher dimensions, Class. Quant. Grav. 32 (2015) 065008 [arXiv:1410.4590] [INSPIRE].

[24] C.M. Harris and P. Kanti, Hawking radiation from a $(4+n)$-dimensional rotating black hole, Phys. Lett. B 633 (2006) 106 [hep-th/0503010] [INSPIRE].

[25] G. Duffy, C. Harris, P. Kanti and E. Winstanley, Brane decay of a $(4+n)$-dimensional rotating black hole: Spin-0 particles, JHEP 09 (2005) 049 [hep-th/0507274] [INSPIRE].

[26] M. Casals, P. Kanti and E. Winstanley, Brane decay of a $(4+n)$-dimensional rotating black hole. II. Spin-1 particles, JHEP 02 (2006) 051 [hep-th/0511163] [INSPIRE].

[27] M. Casals, S.R. Dolan, P. Kanti and E. Winstanley, Brane Decay of a $(4+n)$-Dimensional Rotating Black Hole. III. Spin-1/2 particles, JHEP 03 (2007) 019 [hep-th/0608193] [INSPIRE].

[28] T. Harmark, J. Natario and R. Schiappa, Greybody Factors for d-Dimensional Black Holes, Adv. Theor. Math. Phys. 14 (2010) 727 [arXiv:0708.0017] [INSPIRE].

[29] R.C. Myers and M.J. Perry, Black Holes in Higher Dimensional Space-Times, Annals Phys. 172 (1986) 304 [INSPIRE].

[30] V.P. Frolov and D. Stojkovic, Quantum radiation from a five-dimensional rotating black hole, Phys. Rev. D 67 (2003) 084004 [gr-qc/0211055] [inSPIRE].

[31] A. Ronveaux, Heun's Differential Equations, Oxford University Press, Oxford Oxfordshire (1995). 
[32] J. Meixner and F.W. Schäfke, Mathieusche Funktionen und Sphäroidfunktionen, Springer, Berlin (1954).

[33] J. Meixner, F.W. Schäfke and G. Wolf, Mathieu Functions and Spheroidal Functions and Their Mathematical Foundations (Further Studies), Lect. Notes Math., vol. 837, Springer, Berlin (1980).

[34] P.E. Falloon, P.C. Abbott and J.B. Wang, Theory and computation of spheroidal wavefunctions, J. Phys. A 36 (2003) 5477.

[35] V. Cardoso et al., NR/HEP: roadmap for the future, Class. Quant. Grav. 29 (2012) 244001 [arXiv: 1201.5118] [INSPIRE].

[36] D. Orlando and S.C. Park, Compact hyperbolic extra dimensions: a M-theory solution and its implications for the LHC, JHEP 08 (2010) 006 [arXiv:1006.1901] [INSPIRE].

[37] D.N. Page, Particle Emission Rates from a Black Hole: Massless Particles from an Uncharged, Nonrotating Hole, Phys. Rev. D 13 (1976) 198 [InSPIRE].

[38] S. Mano, H. Suzuki and E. Takasugi, Analytic solutions of the Teukolsky equation and their low frequency expansions, Prog. Theor. Phys. 95 (1996) 1079 [gr-qc/9603020] [INSPIRE].

[39] S. Mano and E. Takasugi, Analytic solutions of the Teukolsky equation and their properties, Prog. Theor. Phys. 97 (1997) 213 [gr-qc/9611014] [InSPIRE].

[40] M. Sasaki and H. Tagoshi, Analytic black hole perturbation approach to gravitational radiation, Living Rev. Rel. 6 (2003) 6 [gr-qc/0306120] [INSPIRE].

[41] G.V. Kraniotis, The Klein-Gordon-Fock equation in the curved spacetime of the Kerr-Newman (anti) de Sitter black hole, Class. Quant. Grav. 33 (2016) 225011 [arXiv: 1602.04830] [INSPIRE]. 